\title{
Viscoelastic Kirchhoff Plate Analysis via Mixed Finite Element Formulation
}

\author{
Fethi Kadığlu and Gülçin Tekin
}

\begin{abstract}
In this study, a functional for the dynamic analysis of viscoelastic Kirchhoff plates is obtained through an efficient systematic procedure based on the Gâteaux Differential Method. For the solution of the derived functional, mixed finite element method in transformed Laplace-Carson space is used. In this functional, there exists four independent variables such as deflection (w), internal forces $\left(M_{x}, M_{y}, M_{x y}\right)$ in addition to the dynamic and geometric boundary condition terms. For modeling the viscoelastic behavior, four parameter solid model is employed. For transformation of the solutions obtained in the LaplaceCarson domain to the time domain, different numerical inverse transform techniques are employed. The developed solution technique is applied to several dynamic example problems for the verification of the suggested numerical procedure.
\end{abstract}

Keywords-dynamic analysis, viscoelastic plates, Gâteaux differential, mixed finite element method, Laplace-Carson transform

\section{Introduction}

The dynamic behavior of viscoelastic plates is of primary importance in engineering applications. Adopting elastic theory to simplify the analysis proves to be inconsistent with reality since most engineering materials exhibit noticeable time effects due to internal friction. Therefore, viscoelastic constitutive relations should be employed instead of elastic constitutive relations to reflect the material behavior. There are many works in the literature on the theory of viscoelasticity [1-2]. For the problems that have complex geometries and constitutive relations, closed form solutions are often not possible and numerical solution methods should be employed. The application of Finite Element Method (FEM) to viscoelastic plate problems has been presented by number of authors [3-6]. When employing the conventional FEM, shear locking is an undesirable phenomenon. Considering suitable mixed finite element methods is a more general way for the prevention of shear locking phenomena. When formulating mixed type finite elements the Hellinger Reissner and the Hu-Washizu variational principles are more popular. However, in this study, the Gâteaux Differential Method, which is more powerful and efficient variational tool when compared to the Hellinger Reissner and the $\mathrm{Hu}-\mathrm{Washizu}$ variational principles, is used to obtain a functional for the dynamic behaviour of viscoelastic Kirchhoff plates in Laplace-Carson space.

Fethi Kadığlu, Gülçin Tekin Istanbul Technical University Turkey
Based on the Gâteaux differential method, Aköz and his co-workers [7-11] analysed the quasi-static and dynamic behaviour of the viscoelastic beam and plate elements by employing the Kelvin and/or Three-parameter Kelvin model. In the solutions, the four parameter Kelvin solid model is employed. The performance of the method is presented by several dynamic example problems.

\section{Field Equations and Functional}

Classical thin plate theory neglects the transverse shear effects and field equations for viscoelastic Kirchhoff plates in Laplace-Carson space are given in (1). For more information, the reader is referred to the study of Aköz et al. [11].

$$
\begin{aligned}
& -\frac{\partial^{2} \bar{M}_{x}}{\partial x^{2}}-\frac{\partial^{2} \bar{M}_{y}}{\partial y^{2}}-2 \frac{\partial^{2} \bar{M}_{x y}}{\partial x \partial y}=\bar{q} \\
& -\bar{M}_{x}-\bar{D}^{\star}\left(\frac{\partial^{2} \bar{w}}{\partial x^{2}}+v \frac{\partial^{2} \bar{w}}{\partial y^{2}}\right)=0 \\
& -\bar{M}_{y}-\bar{D}^{\star}\left(\frac{\partial^{2} \bar{w}}{\partial y^{2}}+v \frac{\partial^{2} \bar{w}}{\partial x^{2}}\right)=0 \\
& -\bar{M}_{x y}-(1-v) \bar{D}^{\star} \frac{\partial^{2} \bar{w}}{\partial x \partial y}=0
\end{aligned}
$$

where $\bar{q}$ represents the normal load distribution in the Laplace-Carson domain, $\bar{M}_{x}, \bar{M}_{y}$ and $\bar{M}_{x y}$ represents the moment resultants in the same space. Positive directions of the stress resultants are shown in Fig. 1. In addition, $\bar{w}$ is the transverse displacement of the plate's middle surface in the Laplace-Carson domain, $v$ is the Poisson's ratio and $\bar{D}^{\star}$ is the operator form of the flexural rigidity of the plate and it is related with the creep function $\bar{Y}^{*}$ as follows:

$$
\bar{D}^{\star}=\frac{h^{3}}{12} \bar{Y}^{\star}
$$

where $h$ is the thickness of the plate. 


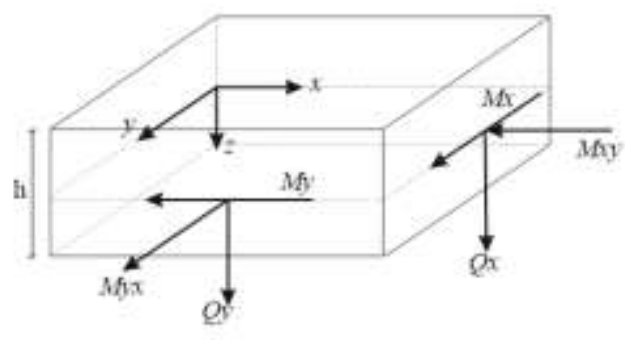

Figure 1. Internal Forces

The boundary conditions in the Laplace-Carson space can be written in symbolic form as below:

$$
\begin{gathered}
\bar{T}-\hat{\bar{T}}=0 \\
-\bar{M}+\hat{\bar{M}}=0 \\
\bar{W}^{\prime}-\hat{\bar{W}}^{\prime}=0 \\
-\bar{W}+\hat{\bar{W}}=0
\end{gathered}
$$

Equation (3) and (4) represent the dynamic and geometric boundary conditions, respectively.

Field equations can be written in operator form as:

$$
\bar{Q}=\bar{L} \bar{y} \cdot \bar{f}
$$

where $\overline{\boldsymbol{Q}}$ is a potential operator if the equality

$$
<d \overline{\boldsymbol{Q}}\left(\overline{\boldsymbol{y}}, \overline{\boldsymbol{y}}^{\prime}\right), \overline{\boldsymbol{y}}^{*}>=<d \overline{\boldsymbol{Q}}\left(\overline{\boldsymbol{y}}, \overline{\boldsymbol{y}}^{*}\right), \overline{\boldsymbol{y}}^{\prime}>
$$

is satisfied [12], where $d \overline{\boldsymbol{Q}}\left(\overline{\boldsymbol{y}}, \overline{\boldsymbol{y}}^{\prime}\right)$ and $d \overline{\boldsymbol{Q}}\left(\overline{\boldsymbol{y}}, \overline{\boldsymbol{y}}^{*}\right)$ are the Gâteaux derivatives of the operator $\overline{\boldsymbol{Q}}$ and the parenthesis indicate the inner products.

The Gâteaux derivative of an operator is defined as:

$$
d \overline{\boldsymbol{Q}}\left(\overline{\boldsymbol{y}}-\overline{\boldsymbol{y}}^{\prime}\right)=\left.\frac{\partial \overline{\boldsymbol{Q}}\left(\overline{\boldsymbol{y}}+\tau \overline{\boldsymbol{y}}^{\prime}\right)}{\partial \tau}\right|_{\tau=0}
$$

where $\tau$ is a scalar quantity. After satisfying the requirement, the functional is obtained as:

$$
I(\overline{\boldsymbol{y}})=\int_{0}^{1}<\overline{\boldsymbol{Q}}(s \overline{\boldsymbol{y}}, \overline{\boldsymbol{f}}), \overline{\boldsymbol{y}}>d s
$$

where $s$ is a scalar quantity. The explicit form of the functional corresponding to the field equations of the viscoelastic Kirchhoff plates in the Laplace-Carson domain becomes:

$$
\begin{aligned}
& I(\bar{y})=\left[\bar{w}_{, x}, \bar{M}_{x, x}\right]+\left[\bar{w}_{y,}, \bar{M}_{y, y}\right]+\left[\bar{w}_{, x}, \bar{M}_{x y, y}\right] \\
& +\left[\bar{w}_{, y}, \bar{M}_{x y, x}\right]-[\bar{q}, \bar{w}]-\frac{1}{2 \bar{D}\left(1-v^{2}\right)}\left\{\left[\bar{M}_{x}, \bar{M}_{x}\right]+\left[\bar{M}_{y}, \bar{M}_{y}\right]\right\} \\
& +\frac{v}{\bar{D}\left(1-v^{2}\right)}\left[\bar{M}_{x}, \bar{M}_{y}\right]-\frac{1}{\bar{D}(1-v)}\left[\bar{M}_{x y}, \bar{M}_{x y}\right] \\
& -[\hat{\bar{T}}, \bar{w}]_{\sigma}-\left[(\bar{M}-\hat{\bar{M}})^{,} \bar{w}^{\prime}\right]_{\sigma}-[\hat{\bar{w}}, \bar{M}]_{\varepsilon}-[(\bar{w}-\hat{\bar{w}}), \bar{T}]_{\varepsilon}
\end{aligned}
$$

Parentheses with the subscripts $\sigma$ and $\varepsilon$ represents the dynamic and geometric boundary conditions, respectively.

In order to derive the finite element formulation, first the interpolation function must be chosen. Rectangular master element as illustrated in Fig. 2 is used in the formulation. The shape functions for rectangular master element are:

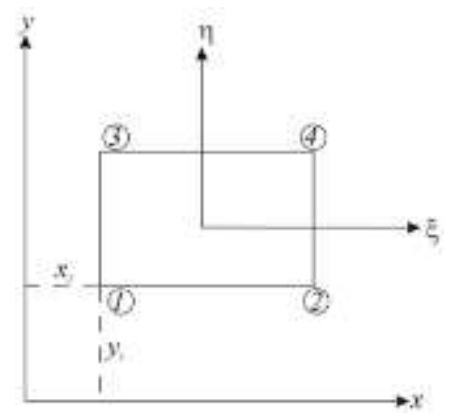

Figure 2. Rectangular master element

$$
\begin{aligned}
& N_{1}=(1-\xi)(1-\eta) \\
& N_{2}=\xi(1-\eta) \\
& N_{3}=(1-\xi) \eta \\
& N_{4}=\xi \eta
\end{aligned}
$$

The four variables of the functional given in (9) are expressed by the shape functions and inserted into (9). After simplifying with respect to nodal variables, the element matrix is derived. The numerical solutions obtained in the Laplace-Carson space are transformed to the time space using different numerical inverse Laplace transform techniques such as MDOP, Dubner \& Abate's and Durbin's methods. Papers for discussion of Laplace inversion techniques, see [13-16].

\section{Numerical Examples}

The plate structure is considered as a four-parameter solid model. Due to the symmetry, the computations are carried out for a quarter of the plate structure.

The material properties are assumed to be Fourparameter solid model (as illustrated in Fig. 3):

$$
\begin{aligned}
& \mathrm{E}_{1}=98 \mathrm{MPa}, \\
& \eta_{1}=98 \mathrm{MPa} . \mathrm{s}, \\
& \mathrm{E}_{2}=274.4 \mathrm{MPa}, \\
& \eta_{2}=274.4 \mathrm{MPa} . \mathrm{s}, \\
& v=0.3
\end{aligned}
$$




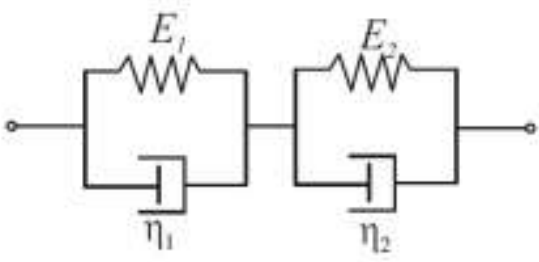

Figure 3. Four-Parameter Solid Model

Hence, the relaxation modulus will be

$$
J_{(t)}=\frac{1}{E_{1}}\left(1-e^{-\frac{E_{1}}{\eta_{1}} t}\right)+\frac{1}{E_{2}}\left(1-e^{-\frac{E_{2}}{\eta_{2}} t}\right)
$$

Considering a simply-supported plate whose thickness $\mathrm{h}=0.1 \mathrm{~m}$, length $\mathrm{a}=4 \mathrm{~m}$ and width $\mathrm{b}=4 \mathrm{~m}$, the dynamic behavior viscoelastic thin plate is analyzed for different time-dependent loadings. For the analysis, $4 \times 4$ mesh size is used. For more information about the most suitable mesh scheme, the reader is referred to the study presented by Aköz et al. [11]. In order to transform solutions from the Laplace-Carson domain to the time domain, the MDOP, Dubner \& Abate and Durbin inverse Laplace transform methods are employed for $\mathrm{aT}=5, \mathrm{~N}=100$ and $\mathrm{T}=20 \mathrm{~s}$. The most suitable values for the effective parameters of the inverse transform methods that decreasing the fluctuation in the solutions, see [11].

The geometry and boundary conditions of the plate structure are shown in Fig. 4. The time histories of loads in numerical examples are illustrated in Fig. 5.

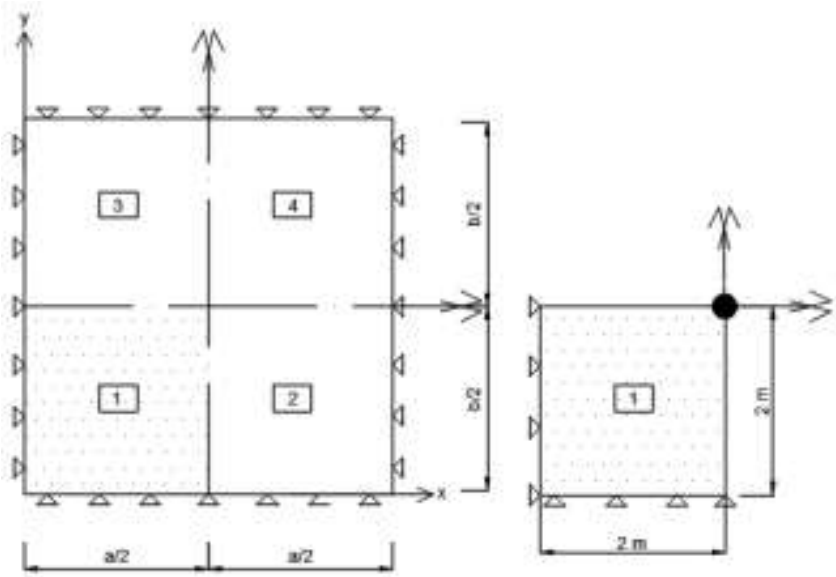

Figure 4. Geometrical properties of the simply supported plate

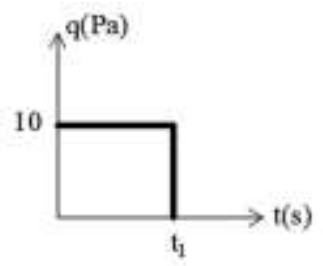

Type I

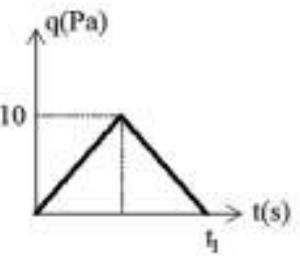

Type II
(Rectangular Impulsive Load) (Triangular Impulsive Load)

\section{Example 1:}

In this example, a simply-supported viscoelastic plate subjected to rectangular impulsive load for $\mathrm{t}_{1}=10 \mathrm{~s}$ (Type I) is considered. For the numerical inversion, MDOP, Dubner \& Abate's and Durbin's methods are employed. The material density $\rho$ is assumed to be $200 \mathrm{~kg} / \mathrm{m}^{3}$ for the dynamic response. The central displacement and bending moment-time variations are presented in Fig. 6 for Dubner \& Abate's and Durbin's methods. Fluctuation is observed in the MDOP inverse transform method for the time-dependent bending moment values at the center of the plate as time increases. In addition, the dynamic behavior of the viscoelastic plate will eventually disappear with time as expected.
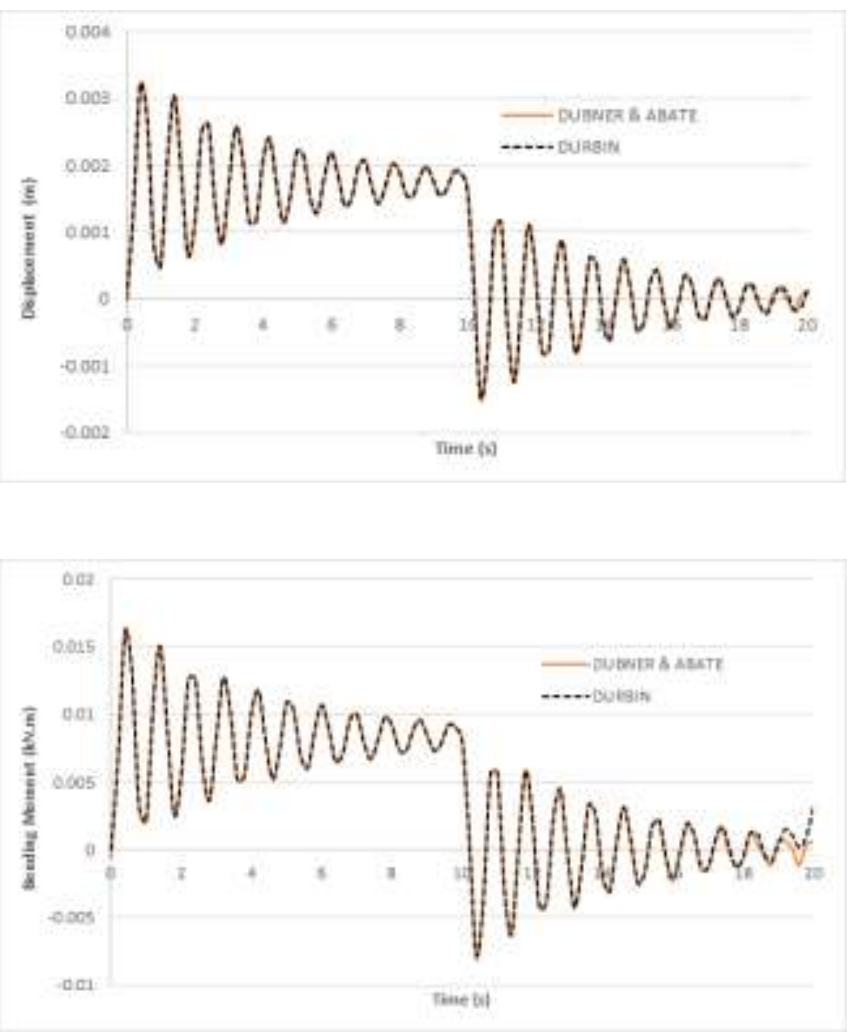

Figure 6. Displacement and Bending Moment-Time variation

\section{Example 2:}

In this example, the effect of the damping ratio $(E / \eta)$ in the dynamic behavior of a simply-supported viscoelastic thin plate is considered. The material density $\rho$ is assumed to be $200 \mathrm{~kg} / \mathrm{m}^{3}$ for the dynamic response. Durbin's inverse transform method' results are presented in Fig. 7 for triangular impulsive load (Type II) for $\mathrm{t}_{1}=10 \mathrm{~s}$. As expected, the vibration behavior of the viscoelastic plate resembles the vibration of an elastic plate for small values of viscosity coefficient. 


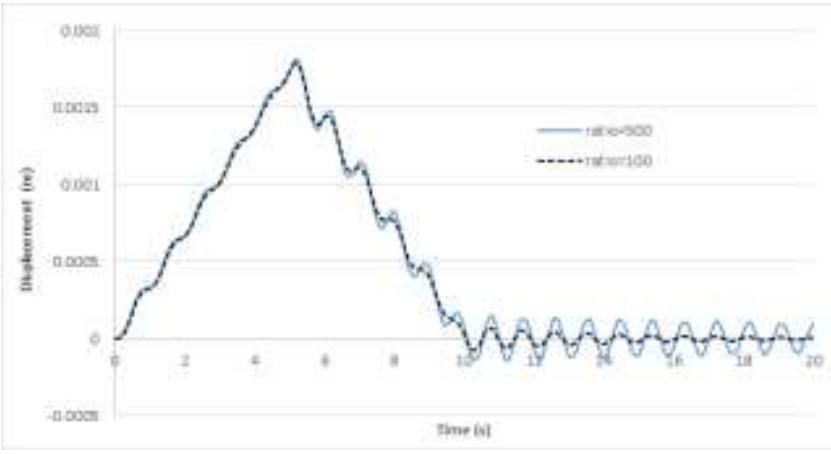

Figure 7. Effect of viscosity coefficient on the amplitude of displacement

\section{Conclusion}

The dynamic behavior of viscoelastic thin plates is analyzed via the mixed finite element formulation in the Laplace-Carson space. The four-parameter solid model is employed in viscoelastic modeling. The functional obtained for the dynamic analysis of viscoelastic thin plates through a systematic procedure based on the Gâteaux differential has four independent variables such as displacement, bending moments and twisting moment in addition to boundary condition terms. For transformation of the solutions obtained in the Laplace-Carson space to the real time space, different numerical inverse Laplace transform techniques such as MDOP, Dubner \& Abate and Durbin are employed. The performance of the developed mixed finite element formulation is tested through several dynamic example problems.

\section{Acknowledgment}

This research is supported by the Scientific and Technological Research Council of Turkey under the grant number 213M332. The authors gratefully acknowledge this support.

\section{References}

[1] W. Flügge, Viscoelasticity, second ed., Springer: Berlin, 1975.

[2] R. M. Christensen, Theory of Viscoelasticity, 2nd ed., Academic Press: New York, 1982

[3] Y. Z. Wang, T. J. Tsai, "Static and dynamic analysis of a viscoelastic plate by the finite element method," Appl. Acoust., vol. 25, pp. 77-94, 1988.

[4] S. Yi, H. H. Hilton, "Dynamic finite element analysis of viscoelastic composite plates,” Int. J. Numer. Meth. Eng., vol. 37, pp. 4081-96, 1994.

[5] M. H. Ilyasov, A. Y. Aköz, "The vibration and dynamic stability of viscoelastic plates," International Journal of Engineering Sciences, vol. 38, pp. 695-714, 2000.

[6] B. Temel, M. F. Sahan, "Transient analysis of orthotropic, viscoelastic thick plates in the Laplace domain," European Journal of Mechanics A/Solids, vol. 37, pp. 96-105, 2013.

[7] A. Y. Aköz, F. Kadığlu, "The mixed finite element method for the quasi-static and dynamic analysis of viscoelastic Timoshenko beams," Int. J. Numer. Meth. Eng, vol. 44, pp. 1909-32, 1999.

[8] F. Kadıŏlu, A. Y. Aköz, "The mixed finite element method for the dynamic analysis of visco-elastic circular beams," Proc. of the 4th International Conference on Vibration Problems, Jadavpur University 1999.
[9] F. Kadığlu, A. Y. Aköz, "The quasi-static and dynamic responses of viscoelastic parabolic beams,"Proc. on the 11th National Applied Mechanics Meeting (in Turkish). Bolu-Turkey, 2000.

[10] F. Kadıŏlu, A. Y. Aköz, "The mixed finite element for the quasistatic and dynamic analysis of viscoelastic circular beams," International Journal of Structural Engineering and Mechanics, vol. 15, pp. 735-752, 2003.

[11] A. Y. Aköz, F. Kadıoğlu, G. Tekin, "Quasi-static and dynamic analysis of viscoelastic plates," Mechanics of Time-Dependent Materials, DOI 10.1007/s11043-015-9274-8, 2015.

[12] J. T. Oden, J. N. Reddy, "Variational Methods in Theoretical Mechanics," Springer-Berlin, 1976.

[13] H. Dubner, J. Abate, "Numerical inversion of Laplace transforms by relating them to the finite Fourier cosine transform," J. ACM., vol. 15,pp. 115-123, 1968.

[14] VI, Krylov, N. S. Skoblya, "Handbook of numerical inversion of Laplace transforms," Translated from Russian, Israel Program for Scientific Translations, Jerusalem, 1969.

[15] F. Durbin, "Numerical inversion of Laplace transforms: An efficient improvement to Dubner and Abate's method," Comput. J., vol. 17, pp. 371-376, 1974.

[16] G. V. Narayanan, D. E. Beskos, "Numerical operational methods for time-dependent linear problems," Int. J. Numer. Meth. Eng., vol. 18, pp. 1829-1854, 1982.

About Author (s):

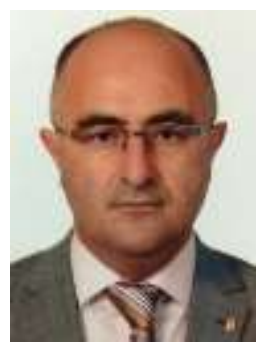

Dr. Kadıŏglu received his BSc, MSc and $\mathrm{PhD}$ from Istanbul Technical University, Istanbul, Turkey. $\mathrm{He}$ is currently working as an Associate Prof. in the Department of Civil Engineering at Istanbul Technical University. He published over 35 papers in international journals and conferences, 5 books and book chapters in the fields of Structural Mechanics. Dr. Kadıoğlu's current research interests include: Composite Structures, Stability of Structures and Vibration, Elastic, Viscoelastic Analysis, Structural Analysis and Finite Element Methods.

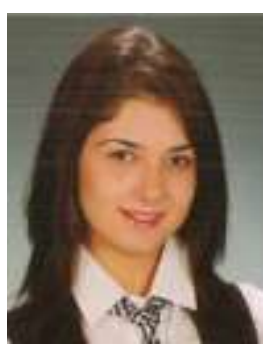

Gülçin Tekin received her BSc degree in Civil Engineering from Yildiz Technical University, Istanbul, Turkey. She completed MSc degree at the Bogaziçi University, Istanbul, Turkey. She is currently a third-year $\mathrm{PhD}$ student in the department of Civil Engineering at the Istanbul Technical University, Istanbul, Turkey. Presently she is working as a research assistant at Istanbul Technical University. Her areas of interest are Structural Analysis, Mixed Finite Element Methods. 